\title{
Glomerulonefrite acuta post-infettiva in un paziente anziano e diabetico con infezione cutanea da Staphylococcus Aureus meticillino resistente
}

\author{
Maria Laura Standoli ${ }^{1}$, Nadia Albasini ${ }^{1}$, Carlo Magarini ${ }^{1}$, Raffaela Sciri ${ }^{1}$, Francesca Trivelli ${ }^{1}$, Carla Covarelli ${ }^{2}$, \\ Rachele Del Sordo ${ }^{2}$, Livio Marchese ${ }^{3}$, Gianrenato Nori ${ }^{1}$ \\ ${ }^{1}$ S.C. Nefrologia e Dialisi, Azienda Ospedaliera di Terni, Terni \\ ${ }^{2}$ S.C. Anatomia e Istologia Patologica, Azienda Ospedaliera Universitaria di Perugia, Perugia \\ ${ }^{3}$ Scuola di Specializzazione Patologia Clinica, Università degli Studi di Perugia, Perugia
}

\begin{abstract}
Acute post-infectious glomerulonephritis in an elderly patient, diabetic with methicillin-resistant staphylococcus aureus cutaneous infection

Post-infectious glomerulonephritis epidemiology is changing, especially in western Countries, probably because of an increase of elderly patients showing more complex comorbidities.

Beta hemolytic Streptococcus represents the main pathogenic agent involved in post-infectious glomerulonephritis in children, while methicillin-resistant Staphylococcus Aureus (MRSA) is prevalent in post-infectious glomerulonephritis in elderly patients.

In adult and elderly patients, post-infectious glomerulonephritis classic features (nephritic syndrome with hematuria, arterial hypertension, edema, renal function impairment) are rarely present and diagnosis could often be missed.

We describe the case of a 71 years old patient; the patient was diabetic and hypertensive and he described the presence of acute kidney injury and severe metabolic acidosis, probably due to metformin treatment.

Although metformin withdrawal and renal replacement therapy were started, renal function did not improve; during the following days nephrotic proteinuria appeared together with progressive diuresis reduction to arrive to oligo-anuria.

Screening for auto-antibodies was negative, while a MRSA was identified in a cultural test performed on an ulcerative wound of the left foot.

Renal biopsy highlighted diffuse proliferative post-infectious glomerulonephritis and steroidal therapy was started with progressive diuresis and renal function recovery.

In adult and elderly patients, renal biopsy acquires an important role in post-infectious glomerulonephritis differential diagnosis, especially in presence of acute renal failure and urinary sediment abnormalities.
\end{abstract}

Keywords: Acute kidney disease, Acute glomerulonephritis, Post-infectious glomerulonephritis, Post-staphylococcal glomerulonephritis

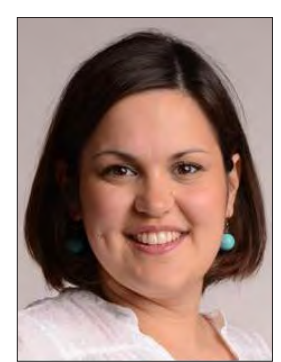

Maria Laura Standoli

\section{Caso clinico}

Paziente di sesso maschile di 71 anni. Anamnesi patologica remota: diabete mellito tipo 2 noto da 18 anni, ipertensione arteriosa sistemica da 20 anni, cerebrovasculopatia e arteriopatia obliterante arti inferiori; in terapia domiciliare con metformina, glimepiride, ASA, atenololo, amlodipina, ramipril + idro-

Accepted: May 5, 2015

Published online: May 11, 2015

Indirizzo per la corrispondenza:

Maria Laura Standoli

Via Battisti 45/E, 05100 Terni

mlstandoli@gmail.com

clorotiazide e allopurinolo. Pregressa normofunzione renale con albuminuria ( $\leq 300 \mathrm{mg} /$ die).

Giungeva alla nostra osservazione per riferita nausea da 4 giorni, singhiozzo incoercibile, anoressia e ipertensione arteriosa non controllata dalla terapia in atto. Al momento del ricovero (17/07/2014), presentava insufficienza renale acuta (IRA) con diuresi conservata (creatinina $6.12 \mathrm{mg} / \mathrm{dL}$, clearance creatinina sec. MDRD $9 \mathrm{~mL} / \mathrm{min}$, diuresi $\geq 1000$ $\mathrm{cc} /$ die), acidosi lattica $\left(\mathrm{pH} 7.12, \mathrm{HCO}_{3}^{-} 11.8 \mathrm{mmol} / \mathrm{L}\right.$, lattati $5.4 \mathrm{mmol} / \mathrm{L}$ ) e ipoglicemia severa (glicemia $40 \mathrm{mg} / \mathrm{dL}$ ). All'esame obiettivo: paziente apiretico, nulla di rilevante all'EO di cuore, torace e addome e lievi segni di disidratazione muco-cutanea; da segnalare un'ulcera a carico della pianta del piede sinistro.

Ipotizzando un'insufficienza renale di origine pre-renale, data l'anamnesi di anoressia e vomito in concomitanza con I'uso di ACE-inibitori e diuretici, il paziente veniva adeguata- 
mente idratato. In considerazione dell'acidosi lattica secondaria a metformina, venivano sospesi gli ipoglicemizzanti orali e veniva introdotta insulina al bisogno. Veniva quindi iniziato il trattamento dialitico.

L'ecografia renale evidenziava reni di normali dimensioni con corticale ispessita e assenza della fase diastolica all'ECD. All'esame chimico-fisico delle urine: proteine $100 \mathrm{mg} / \mathrm{dL}$, emoglobina $1 \mathrm{mg} / \mathrm{dL}$; al sedimento: batteri, leucociti e 10-20 emazie per campo che all'esame a contrasto di fase risultavano dismorfiche con una percentuale di acantociti $>5 \%$; non segnalati cilindri. Agli esami ematici: leucocitosi neutrofila (GB 15910 mmc; neutrofili 87\%), anemia (Hb 10.8 g/dL), PTHi 144 $\mathrm{pg} / \mathrm{mL}$; incremento delle componenti $\alpha 1$ e $\alpha 2$ all'elettroforesi proteica, incremento di VES e PCR $(55 \mathrm{~mm} / \mathrm{h}$ e $14.66 \mathrm{mg} / \mathrm{dL}$, rispettivamente); C3: $81 \mathrm{mg} / \mathrm{dL}$ (VN 70-170 mg/dL), C4:22 mg/ dL (VN 16-45 mg/dL); IgG: 681 mg/dL; IgA: 284 mg/dL; IgM: $45 \mathrm{mg} / \mathrm{dL}$; ANCA, ANA, Ab anti-ENA, fattore reumatoide, titolo antistreptolisinico negativi, debole positività per $A b$ anti membrana glomerulare, marker epatite B e C negativi e proteinuria di Bence Jones negativa.

Alla radiografia del piede, riscontro di erosioni ossee del IV metatarso e tampone colturale dell'ulcera cutanea positivo per Staphylococcus Aureus meticillino resistente; veniva avviata una terapia antibiotica inizialmente con fluorochinoloni (20/07) e successivamente con linezolid e tigeciclina (1/08) sulla base dell'antibiogramma.

Alla TC del torace si evidenziava la comparsa di un versamento pleurico bilaterale; non c'erano altre alterazioni significative.

Dopo 6 giorni di ricovero, a fronte di una normalizzazione dell'equilibrio acido-base, si assisteva a una contrazione della diuresi fino all'anuria; veniva, pertanto, proseguita la terapia dialitica.

In data 30/07/2014 veniva effettuata una biopsia renale che evidenziava alla microscopia ottica 13 glomeruli di cui 4 in sclero-ialinosi; i rimanenti mostravano ipercellularità con spiccata proliferazione endocapillare associata a infiltrazione di granulociti neutrofili e con notevole riduzione dei lumi capillari; presenza di aderenze capsulari, in assenza di semilune. Nell'interstizio, presenza di focolai di infiltrato infiammatorio cronico, con presenza di granulociti neutrofili, associati ad atrofia tubulare e fibrosi. Nei limiti della norma i vasi valutabili (Figg. 1,2). All'immunofluorescenza, presenza di C3 con aspetto a cielo stellato (Figg. 3, 4) e depositi parietali periferici di IgA. Tali reperti, in considerazione dei dati anamnestici, risultavano compatibili con glomerulonefrite proliferativa diffusa post-infettiva a depositi di IgA.

Dopo 12 giorni di terapia antibiotica mirata con miglioramento del quadro infettivo locale e degli indici di flogosi, in considerazione della scarsa risposta in termini di funzione renale, veniva avviata una terapia steroidea con 3 boli di metilprednisone seguito da prednisone per via orale.

Rapidamente si assisteva a una progressiva ripresa della diuresi e al graduale miglioramento della funzione renale, tale da rendere possibile la sospensione della dialisi, pur per-

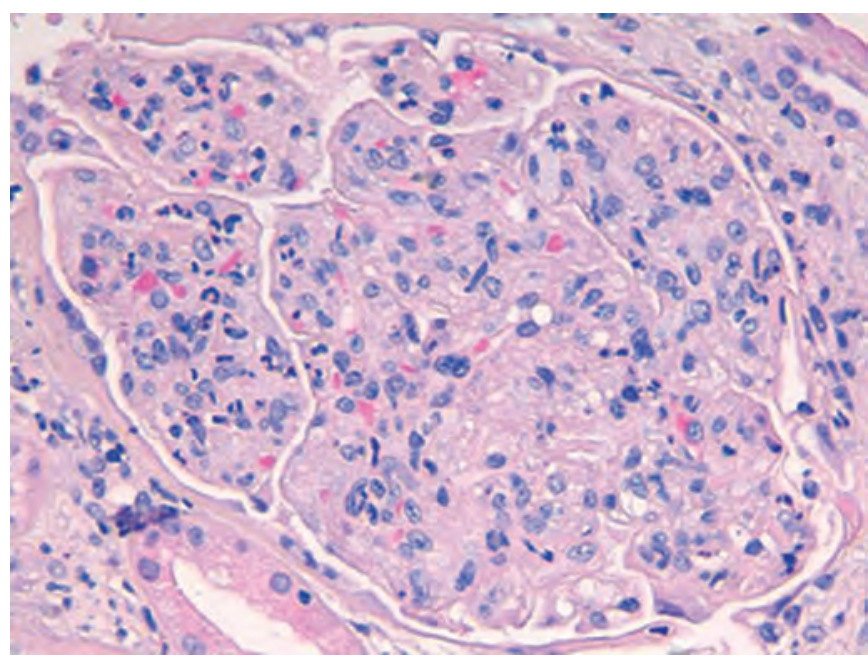

Fig. 1 - Ematossilina eosina 40 ×; presenza di granulociti, proliferazione endocapillare con restringimento del lume dei capillari.

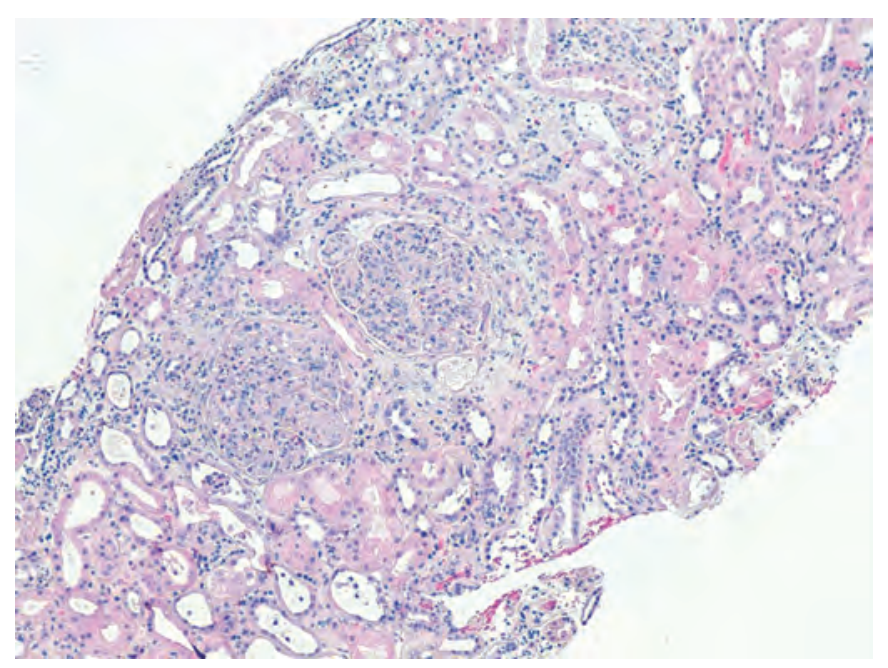

Fig. 2 - Ematossilina eosina $20 x$; due glomeruli con proliferazione endocapillare, con aderenze capsulari in assenza di semilune.

sistendo insufficienza renale di grado moderato e proteinuria in range nefrosico $(5.6 \mathrm{~g} / 24 \mathrm{~h})$.

II paziente veniva dimesso il 27/08/2014 con terapia steroidea orale a scalare, insulina ai pasti, furosemide, atenololo, amlodipina, ramipril, darbepoetina, folina e calcio acetato.

Il controllo a distanza di 20 giorni dalla dimissione mostrava creatinina $2.2 \mathrm{mg} / \mathrm{dL}$, azotemia $70 \mathrm{mg} / \mathrm{dL}$, proteinuria 2 $\mathrm{g} / 24$ ore ed emoglobina $10 \mathrm{mg} / \mathrm{dL}$ (dopo 2 trasfusioni di globuli rossi concentrati).

\section{Discussione}

L'associazione tra insufficienza renale e infezione è conosciuta fin dalla metà del 1800 e lo Streptococco beta emo- 


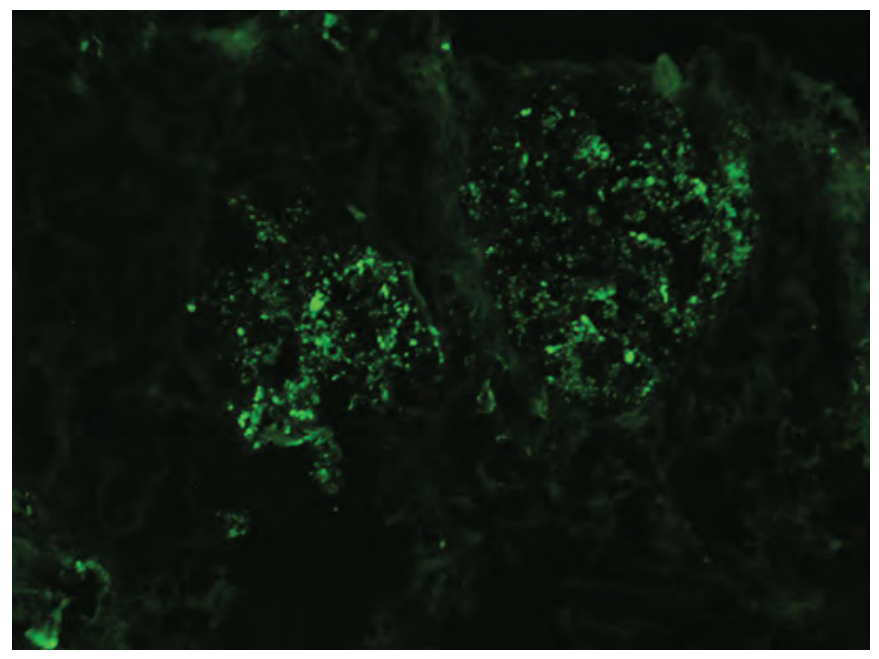

Fig. 3 - Immunofluorescenza per C3 20x; presenza di due glomeruli con pattern a cielo.

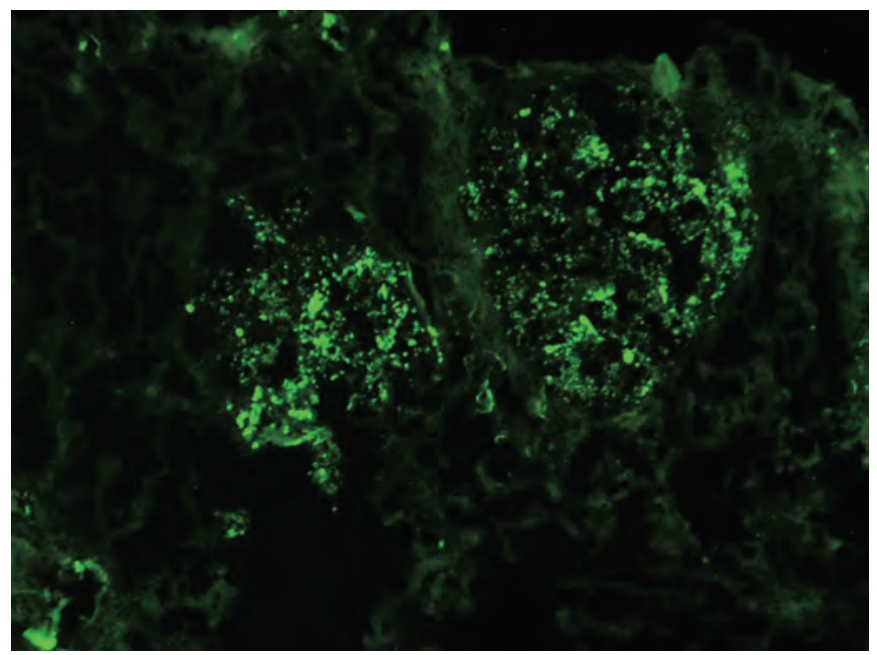

Fig. 4 - Immunofluorescenza per C3 20x; presenza di due glomeruli con pattern a cielo stellato.

litico era identificato come patogeno più frequente nella glomerulonefrite acuta post-infettiva (GNAPI).

La presentazione clinica classica è caratterizzata da sindrome nefritica con ematuria, proteinuria, ipertensione arteriosa, edemi declivi e vario grado di insufficienza renale (1). Sebbene la GNAPI sia una malattia tipica dell'età pediatrica, è stata riscontrata anche nei soggetti adulti e anziani, spesso correlata ad altri agenti infettivi, in particolare, soprattutto nei Paesi industrializzati, allo Staphylococcus Aureus meticillino resistente (MRSA). II decorso clinico della GNAPI da MRSA può essere caratterizzato da glomerulonefrite rapidamente progressiva con proteinuria in range nefrosico ed è responsabile di un quadro morfologico con depositi di IgA (2).

$\mathrm{Se}$, nei pazienti pediatrici, il sito di infezione più frequente è rappresentato dalle vie aeree superiori, nei pazienti anziani sono più frequenti infezioni cutanee ( $28 \%$ dei casi), che includono anche le infezioni della ferita chirurgica, infezioni polmonari (16\%) e infezioni delle vie urinarie (13\%); nell'anziano, lo Stafilococco, come già detto, è il germe più comunemente coinvolto (46\% dei casi), seguito dallo Streptococco (16\%) e da non usuali batteri Gram-negativi (3-5).

Il periodo di latenza tra l'infezione delle vie aeree superiori e l'insorgenza di insufficienza renale è, nel bambino, tipicamente di 1-6 settimane. Spesso, nel paziente anziano, l'infezione viene scoperta al momento dell'insorgenza dell'insufficienza renale, a indicare che o non c'è tempo di latenza tra infezione e nefrite o che, più spesso, l'infezione rimane misconosciuta per molto tempo $(3,5,6)$. Infatti, segni e sintomi di infezione nel paziente anziano possono essere aspecifici e la febbre, segno cardine dell'infezione nel giovane adulto, è spesso assente nell'anziano $(3,7)$

Se la prognosi nel bambino è generalmente favorevole, nell'adulto e nell'anziano è più severa $(8,9)$ : il $25 \%$ dei pazienti adulti ha necessità di dialisi alla diagnosi e il recupero completo della funzione renale avviene nel $40-50 \%$ dei pazienti; negli altri rimane un sedimento urinario attivo, ipertensione arteriosa e il $10-60 \%$ dei pazienti, a seconda delle casistiche, sviluppa insufficienza renale cronica terminale $(10,11)$.

Secondo alcuni Autori, questo potrebbe derivare da una maggiore suscettibilità alle infezioni negli anziani per alterazioni del sistema immune legate sia a fenomeni di immuno-senescenza che ad alterazioni secondarie a condizioni particolari, più frequenti nell'anziano, come la malnutrizione o le comorbidità: il diabete mellito è la condizione maggiormente predisponente alle infezioni, seguito dalle neoplasie maligne (12).

Al contrario, fattori prognostici favorevoli circa l'evoluzione sono giovane età, creatininemia nella norma al momento della biopsia, assenza di nefropatie concomitanti e assenza di infiammazione interstiziale (13).

Nel nostro caso, al momento dell'ammissione in ospedale, la causa dell'insufficienza renale acuta sembrava tutt'altra, ma all'idratazione, alla sospensione dell'ipoglicemizzante orale e alla correzione del quadro di acidosi mediante trattamento dialitico faceva seguito la contrazione della diuresi, fino all'anuria, con comparsa di segni di ritenzione idro-salina; pertanto dovevano essere prese in considerazione altre ipotesi diagnostiche.

II paziente, diabetico con complicanze micro e macroangiopatiche, presentava un'ulcera cutanea infetta: l'incremento degli indici di flogosi e la presenza di MRSA all'esame colturale del tampone cutaneo facevano porre il sospetto diagnostico di insufficienza renale acuta correlata all'infezione, sebbene non fosse presente ipocomplementemia, marker laboratoristico fortemente suggestivo di GNAPI, presente in circa il 70\% dei casi.

In considerazione dell'età del paziente, delle condizioni cliniche generali, delle comorbidità, ma, soprattutto, della sua storia clinica di diabetico, vasculopatico con insufficienza 
renale rapidamente progressiva, è stato ritenuto opportuno effettuare un accertamento istologico che ha portato alla diagnosi di glomerulonefrite proliferativa diffusa post-infettiva a depositi di IgA.

La presenza di IgA all'immunofluorescenza è un'evenienza segnalata in letteratura (12).

In particolare nei pazienti con nefropatia diabetica a cui si sovrappone la glomerulonefrite acuta post-stafilococcica, si può osservare una dominante o codominante deposizione di IgA a livello mesangiale (14). Questa deposizione potrebbe essere spiegata con un aumento dei livelli sierici di questa classe di immunoglobuline, conseguente a un'aumentata produzione o a una ridotta clearance epatica (12).

In presenza di un'infezione, in particolar modo da Stafilococco, l'osservazione di un'ipocomplementemia agli esami ematici e di un quadro istologico caratterizzato da una proliferazione endocapillare con infiltrazione di neutrofili e da depositi tipo hump al microscopio elettronico fa ritenere più probabile una diagnosi di glomerulonefrite post-infettiva con depositi dominanti di IgA piuttosto che una diagnosi di glomerulonefrite primitiva da depositi di IgA (13).

La prognosi renale dei pazienti anziani con GNAPI sovrapposta a nefropatia diabetica è peggiore di quella di pazienti con lo stesso tipo di glomerulonefrite ma senza nefropatia diabetica sottostante (3). II nostro paziente veniva trattato con terapia antibiotica mirata da 12 giorni, con miglioramento del quadro infettivo locale e riduzione degli indici di flogosi; proseguiva una terapia mirata al controllo della sindrome nefritica e la terapia di supporto della funzione renale. Per il persistere dell'anuria, pur non essendoci una chiara indicazione in letteratura all'utilizzo di una terapia immunosoppressiva (13), valutando attentamente rischi e benefici, in presenza di un processo infettivo in risoluzione, abbiamo deciso di effettuare un tentativo con la terapia steroidea, con ripresa della diuresi e parziale recupero della funzione renale.

L'epidemiologia della glomerulonefrite acuta post-infettiva sta cambiando, soprattutto in considerazione dell'invecchiamento della popolazione e della presenza di pazienti sempre più fragili e con pluripatologie (14). Nel paziente anziano con insufficienza renale acuta e sedimento urinario attivo dovrebbe essere sempre presa in considerazione, tra le diagnosi differenziali, la glomerulonefrite post-infettiva. Data la presentazione e l'evoluzione spesso atipiche, nel paziente adulto-anziano la biopsia renale ha un peso molto importante nell'identificare questo tipo di nefropatia.

In un paziente diabetico complicato con decorso atipico caratterizzato da insufficienza renale rapidamente progressiva, proteinuria in range nefrosico ed ematuria glomerulare e mancato recupero della funzione renale dopo terapia an- tibiotica, la biopsia renale risultava mandatoria e ci ha consentito di chiarire la diagnosi e di effettuare una terapia con esito positivo.

Una diagnosi corretta può portare, tra l'altro, a una più accurata ricerca di eventuali siti infettivi occulti e permettere una cura efficace del paziente.

\section{Disclosures}

Financial support: No financial support was received for this submission.

Conflict of interest: The authors have no conflict of interest.

\section{Bibliografia}

1. Nasr SH, Markowitz GS, Stokes MB, Said SM, Valeri AM, D'Agati VD. Acute post infectious glomerulonephritis in the modern era: experience with 86 adults and review of the literature. Medicine. 2008;87:21-32.

2. Montseny JJ, Meyrier A, Kleinknecht D, Callard P. The current spectrum of infectious glomerulonephritis: experience with 76 patients and review of the literature. Medicine. 1995;74:63-73.

3. Nasr SH, Fidler ME, Valeri AM, et al. Postinfectious glomerulonephritis in the elderly. J Am Soc Nephrol. 2011;22: 187-95.

4. Moroni G, Pozzi C, Quaglini S, et al. Long term prognosis of diffuse proliferative glomerulonephritis associate with infections in adults. Nephrol Dial Transplant 2002;17:1204-11.

5. Keller CK, Andrassy K, Waldherr R, Ritz E. Postinfectious glomerulonephritis--is there a link to alcoholism? Q J Med. 1994;87:97-102.

6. Nasr SH, Radhakrishnan J, D’Agati VD. Bacterial infection-related glomerulonephritis in adult. Kidney Int. 2013;83:792-803.

7. Norman DC. Fever in the Ederly. Clin Infect Dis. 2000;31:148-51.

8. Stratta P, Musetti C, Barreca A, Mazzucco G. New trends of an old disease: the acute post infectious glomerulonephritis at the beginning of the new millenium. J Nephrol. 2014;27(3): 229-39.

9. Coppo R, Gianoglio R, Porcellini MG, Maringhini S. Frequency of renal diseases and clinical indications for renal biopsy in children (Report of the Italian National Registry of Renal Biopsies in Children). Nephrol Dial Transplant. 1998;13:293-7.

10. Hoy WE, White AV, Dowling A. Post-streptococcical glomerulonephritis is a strong risk factor for chronic kidney disease in later life. Kidney Int. 2012;81(10):1026-32.

11. Nast CC. Infection-related glomerulonephritis: changing demographics and outcomes. Adv Chronic Kidney Dis. 2012;19 (2): 68-75.

12. Nasr SH, Markowitz GS, Whelan JD, et al. IgA-dominant acute poststaphylococcal glomerulonephritis complicating diabetic nephropathy. Hum Pathol. 2003;34:1235-41.

13. Cagnoli L, Rigotti A, Grimaldi D. Le glomerulonefriti parainfettive. Giornale Italiano di Nefrologia. 2012;29(Suppl. 56):S28-34.

14. Hirayasu Kai, Yoshio Shimizu, Masahiro Hagiwara, et al. PostMRSA infection glomerulonephritis with marked Staphylococcus aureus cell envelope antigen deposition in glomeruli. J Nephrol. 2006;19:215-9. 


\section{TEST DI VERIFICA}

1) Quanti pazienti hanno necessità di una terapia sostitutiva renale alla diagnosi di glomerulonefrite acuta postinfettiva?
a) $10 \%$
b) $25 \%$
c) $50 \%$
d) $70 \%$

2) Quali sono i patogeni più frequentemente implicati nella patogenesi della glomerulonefrite acuta post-infettiva dell’anziano?
a) Streptococco beta emolitico
b) Staphylococcus Aureus
c) Pseudomonas
d) Haemophilus Influenzae

3) Qual è il sito di infezione più frequente nel paziente anziano?
a) Polmone
b) Cute
c) Vie urinarie
d) Vie biliari

Le risposte corrette alle domande sono pubblicate in questo numero alla pagina successiva. 